\title{
Inhibiting Activity of HIV-1: Protease, Reverse Transcriptase and Integrase All Together by Novel Compounds Using Computational Approaches
}

\author{
Umesh Kalathiya*, Monika Padariya* \\ Department of Pharmaceutical Technology and Biochemistry, Faculty of Chemistry, Gdansk University of \\ Technology, Narutowicza St 11/12, 80-233 Gdansk, Poland.
}

*Corresponding author. email: umesh.kalathiya@pg.gda.pl

Manuscript submitted July 11, 2014; accepted September 20, 2014.

doi: 10.17706/ijbbb.2014.4.6.448-457

\begin{abstract}
Acquired immunodeficiency syndrome (AIDS), caused by human immunodeficiency virus type 1 (HIV-1) infection, is one of the most challenging diseases in recent decades. Nevertheless the shortcomings of chemical drugs such as toxicity, lack of curative effects, the search for more potent anti-HIV agents have been focused in our study. In current study, novel scaffold was designed to having a benzyl and imidazole in it which are very important functional groups to available HIV-1 inhibitors. Based on our novel scaffold, different compounds were designed by adding functions groups at $\mathrm{R}_{1}$ and $\mathrm{R}_{2}$ ends which taken from some FDA approved inhibitors (a reverse design approach). Designed ligands were tested individually with HIV-1 protease, reverse transcriptase and integrase enzymes. Flexible and rigid docking approaches were applied to all complexes and comparative analysis of results was done. Compound C0M5 containing functional groups pyridyl methyl piperazine with acetamide at $\mathrm{R}_{1}$ and indanol at $\mathrm{R}_{2}$ end, and compound C0M8 having cyclopentenopyridine with $\mathrm{N}$-methylacetamide at $\mathrm{R}_{1}$ and octahydro- $1 \mathrm{H}$-isochromeneare at $\mathrm{R}_{2}$ end has shown potential bindings with HIV-1 enzymes. Outcome of proposed work suggests that, designed compound performed as the multifunctional ligands and has the tendency to interact with integrase, reverse transcriptase and integrase with efficient bindings and can be considered as potential inhibitors of HIV-1 enzymes for further testing.
\end{abstract}

Key words: HIV-1, protease, reverse transcriptase, integrase, docking.

\section{Introduction}

Human Immunodeficiency Virus type-1 (HIV-1) is a lentivirus, it belongs to the retrovirus family which is a slow, progressive, and degenerative and induces a life threatening disease for human immune system and is called as the acquired immunodeficiency syndrome (AIDS) [1], [2]. For completing viral replication cycle, HIV-1 requires three different enzymes: (i) Protease (PR) (ii) Reverse Transcriptase (RT) and (iii) Integrase (IN) [3], these enzymes have their specific roles for completing the life cycle of this virus. HIV-1 reverse transcriptase is involved in transcribing RNA of the HIV-1 virus in to DNA, after the generation of viral DNA, it is ejected into the host genome by HIV-1 integrase enzyme [3], [4].

Completion of tasks by reverse transcriptase and integrase will be then followed by HIV-1 protease enzyme (a member of aspartyl family) [5]. Expression of the virus by the host cell produces Gag and Gag-pol proteins [2], HIV-1 protease is very important for viral particle maturation as it cleaves the viral precuros polypetides Gag and Gag-Pol into mature structure and ezymatic proteins [6], [7], which states 
that polyprotein processing is carried out by virus itself and not by the host cell [2].

Understanding functionalities of HIV-1 enzymes, it explains that blocking activity of any one enzyme will block the activity of HIV-1 virus (with possibilities to acquire resistance towards drug over time), and furthermore if activity of all these enzymes (protease, reverse transcriptase and integrase) blocked together will result in not allowing the virus to resist against the drug. In current work, an effort was made to inhibit the activity of all three enzymes with an approach of single drug for different enzyme (multifunctional ligands). Inhibitor designing have proved to be a successful stories from decades and many inhibitors are designed for specific enzymes of HIV-1. But from several reasons such as high-intrinsic toxicity of compounds and highly active resistant viral strains of HIV-1 makes them difficult to sustain their activeness towards the HIV-1 [8]-[10]. So, we propose to target all enzyme together by multifunctional ligands which will result into a better solution for curing acquired immunodeficiency syndrome (this multifunctional ligand approach has been tested before [11], [12]).

HIV-1 protease contains a homo-dimer structure, with 99 residues in each monomer and each monomer is identical to each other, they form a symmetric dyad [13]. Protease enzyme structure is dominated by $\beta$-strands and it has only one $\alpha$-helix [14]. Structures of protease [14], reverse transcriptase [15] and structure of C-terminal and catalytic core domain (directly involved in DNA binding) of integrase enzyme [16] are considered in this study for different docking approaches (Different experiment shows that retroviral integrase enzyme posses three domains, N-terminal, catalytic core and C-terminal domain [16], [17])

\subsection{Available Inhibitors}

The food and Drug Administration (FDA) has approved different antiviral agents to inhibit the HIV-1 enzymes, which includes indinavir, saquinavir, ritonavir, amprenavir, atazanavir, tipranavir and several others under clinical trial [18]. Considering the rapid evolution of drug-resistant variants results in short-lived drugs designed for clinical care, there is an urgent need to develop antiretroviral drugs for current and future wild-type and mutant strains of HIV-1 enzymes. Three key enzymes of HIV1, reverse transcriptase (RT), protease (PR) and integrase (IN) were investigated in this study. Nine different compounds were designed considering the importance of functional groups of already available commercial drugs indinavir, saquinavir, ritonavir, amprenavir and nelfinavir.

\subsection{Structure-Based Design Approach}

An attractive approach in the design of HIV-1 inhibitors has been the use of heterocyclic ring systems to attenuate pharmacokinetic properties, increase binding affinity, and provide novel chemical scaffolds to oppose drug-resistant viral strains. Such scaffolds offer many desirable properties that make them important in molecular design. Heterocycles itself may directly bind to the enzyme forming hydrogen bonds or hydrophobic interactions which results into increased potential [19]. It has been well established that the heterocyclic rings will play a major role in HIV-1 inhibitors due to their conformational restriction as well as their ability to fill hydrophobic pockets and provide functionality for hydrogen bonding interactions [19].

Novel (main) scaffold of this work was designed considering the importance of benzyl and imidazole groups to the HIV-1 enzymes inhibitors. Reverse design approach was applied to design new compounds, instead of taking an already available inhibitor and adding functional groups to it to improve its potency, a novel new scaffold was designed and important functional groups were taken from some of the already approved compound by FDA and they were added in designed scaffold. Designed scaffold is modified at $\mathrm{R}_{1}$ and $\mathrm{R}_{2}$ positions by adding potential functional groups of FDA approved drugs to improve its binding affinity towards selected key enzymes of HIV-1 (Table 1). 


\section{Methodology}

Drug development is a costly and time consuming process through experimental methods, thus molecular docking using computational approaches may reduce time and costs and in recent years. It has been considered as one of significant approach to design and understand drugs at molecular level. In this study, two different docking approaches, flexible and rigid docking were applied and comparative analysis of obtained results was performed. Molecular modeling approach was used to design, minimize and optimize the compounds, initially compounds were created by using Accelrys Discovery Studio Client 3.1 package, Charmm forcefield was applied, Partial Momany Rone charges were added [20], [21].

Table 1. Novel Compounds Designed Based on Benzyl and Imidazole Groups

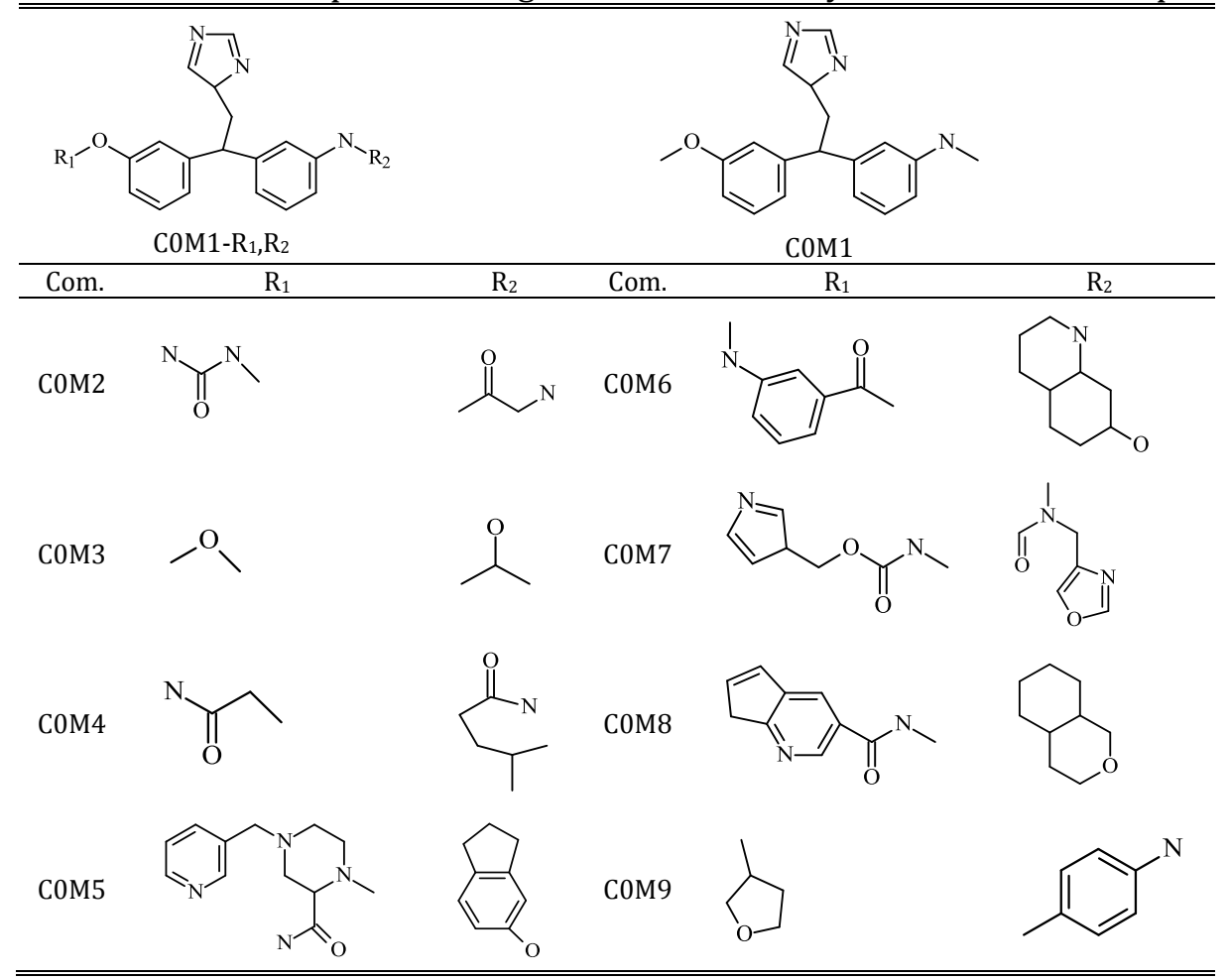

Fig. 1, of (A), (B) and (C) are the active site residues of HIV-1 enzymes, and they are used for performing flexible docking by using CDOCKER (Discovery Studio Client 3.1). These active site residues for each enzyme are the following: (i) HIV-1 protease (Arg8, Leu23, Asp25, Gly27, Ala28, Asp29, Asp30, Gly48, Gly49, Ile50, Pro81, Ile84) [12], [14] (ii) HIV-1 reverse transcriptase (Arg72, Val108, Leu109, Asp110, Val111, Gly112, Asp113, Ala114, Tyr115, Glu151, Met184, Asp185, Asp186, Lys219, His221) [12], [15] and (iii) HIV-1 integrase (Gln62, Asp64, Cys65, Thr66, His67, Glu92, Asp116, Glu138, Gly140, Gln148, Glu152, Asn155, Lys156, Lys159) [16]. And Fig. 1 (D), (E) and (F) are the monomer structures of the enzymes, they are used to perform rigid docking by using AutoDock software package, monomer here are considered for each enzyme respectively to have specific binding towards each monomer of the enzyme and to investigate interactions at molecular aspects.

\subsection{CDOCKER (Discovery Studio Client 3.1)}

Flexible docking was performed by using CDOCKER (CHARMm based DOCKER) [22], in which protein as well as ligand is kept flexible (including bond, angles and dihedrals). In this process proteins and ligand both changes its conformations and tries to fit with each other. CHARMm based DOCKER by Discovery Studio Client 3.1 uses Charmm forcefield and it is a grid based on molecular dynamics docking algorithm 
[22].

CDOCKER includes generation of several random ligand conformations inside active site of target proteins which is then followed by molecular dynamics based simulated annealing involving cooling and heating stages, final refinement is performed by energy minimization [22]. Inputs for these methods were set as: heating ligand up to $700 \mathrm{~K}$ and cooling till $300 \mathrm{~K}$ for $2 \times 10^{3}$ and $5 \times 10^{3}$ steps respectively, and the grid extension was set to eight angstroms. Output obtained was the ten best ranked conformations showing interaction energies towards HIV-1 enzyme.

\subsection{AutoDock}

Rigid docking of protein with ligand was performed by AutoDock software packages, in rigid docking protein was kept as rigid and the ligand was kept flexible (changing its conformations). AutoDock was used for evaluating the conformers and calculating binding free energy of receptor-ligand interactions [23]. Procedure of AutoDock to calculate the interaction of receptor and ligand is divided into three steps: AutoTors, AutoGrid and AutoDock [24], [25].
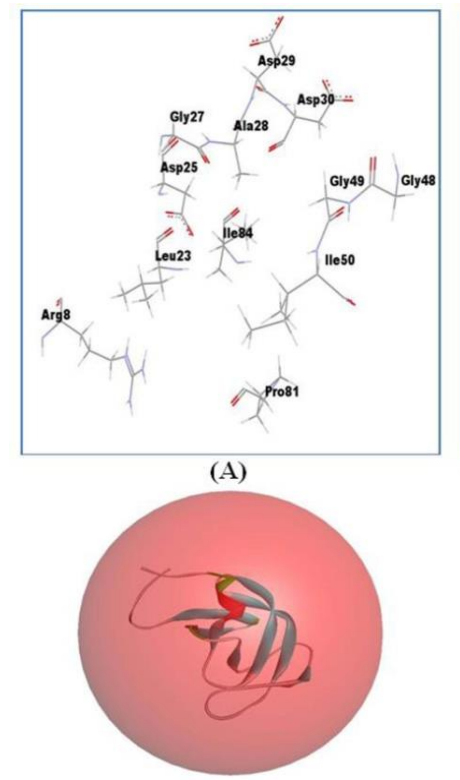

(D)
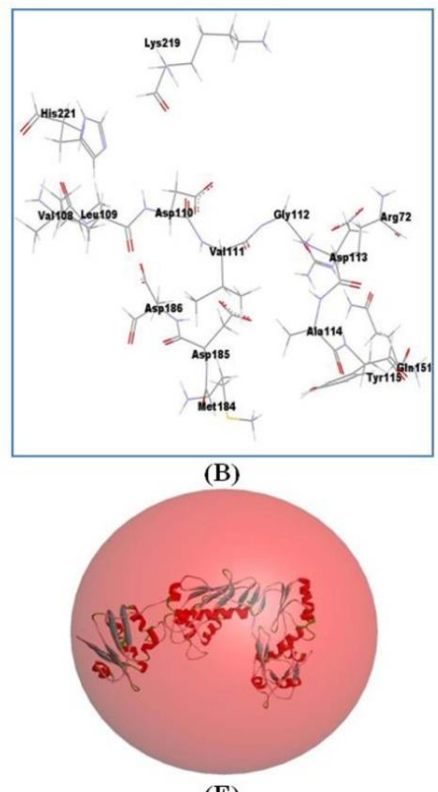

(E)
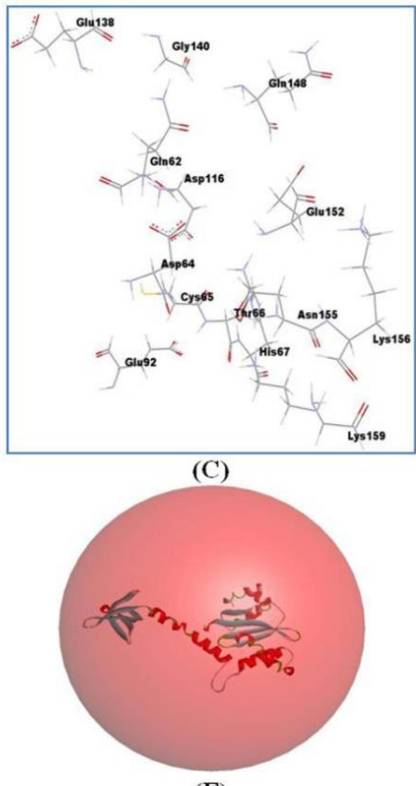

(F)

(IN)

Fig. 1. Active site residues of HIV-1 enzymes for CDOCKER (flexible docking) and monomer of proteins structure for AutoDock (rigid docking). (A) Active site of HIV-1 protease; (B) Active site of HIV-1 reverse transcriptase; (C) Active site of HIV-1 integrase; (D) Monomer of HIV-1 protease (PDB ID: 4LL3 [14]); (E) Monomer of HIV-1 reverse transcriptase PDB ID: 3KLF [15]; (F) Monomer of HIV-1 integrase PDB ID: 1EX4 [16]. (Color scheme: carbon atoms are represented in gray color, oxygen atoms in red and hydrogen atoms in white, $\alpha$-helix in red $\beta$-strands in blue color).

Parameter files for grid calculation as well as for docking calculation were prepared with MGLTools, a graphical user interface for AutoGrid and AutoDock program. For evaluating binding energies of protein ligand complex, three dimensional grid boxes were created by using AutoGrid Algorithm. Grid size was set to $(50 \times 50 \times 50) \AA$ for protease enzyme, $(90 \times 90 \times 120) \AA ̊$ for reverse transcriptase enzyme and $(60 \times 90 \times$ 100) $\AA$ for integrase enzyme in $(x, y, z)$ directions with a common spacing of $1.0 \AA$. Lamarckain genetic algorithm [26] was applied to docking platform/protocol, and the protocol consists of 200 independent runs over 250 population size, maximum energy evaluation of $75 \times 10^{6}$ and number of generation of $27 \times$ $10^{3}$. 


\section{Results and Discussions}

Understanding the importance of the designed scaffold (COM1), it was tested for desired approaches (rigid and flexible docking) with HIV-1 protease, reverse transcriptase and integrase enzyme. Obtained results have shown a quite relevant and comparatively significant activity in all docking approaches with HIV-1 enzymes. Adding functional groups at $\mathrm{R}_{1}$ and $\mathrm{R}_{2}$ ends of C0M1 compound resulted into increased potency of the compound to interact with HIV-1 enzymes. Compounds which were designed based on retrieving functional groups from some already available inhibitors and adding those functional groups to our proposed scaffold (COM5, COM6, COM7, COM8 and C0M9), showed good results in both flexible and rigid docking and they could be considered as the best compound based on their free binding energies they obtained towards HIV-1 enzymes (Table 2).

Table 2. Binding/Interaction Energies for Receptor-Ligand Interaction Obtained from Flexible and Rigid Docking (Autodock and CDOCKER results)

\begin{tabular}{|c|c|c|c|c|c|c|c|c|c|}
\hline \multirow[t]{3}{*}{$\begin{array}{l}\text { Com. } \\
\text { No. }\end{array}$} & \multirow{2}{*}{\multicolumn{3}{|c|}{$\begin{array}{c}\text { CDOCKER Results } \\
\text { (flexible docking) }\end{array}$}} & \multicolumn{6}{|c|}{$\begin{array}{l}\text { AutoDocK Results } \\
\text { (rigid docking) }\end{array}$} \\
\hline & & & & \multicolumn{2}{|c|}{$\begin{array}{l}\text {-Lowest Binding } \\
\text { Energy }(\mathrm{kcal} / \mathrm{mol})\end{array}$} & \multicolumn{4}{|c|}{$\begin{array}{c}\text {-Mean Binding } \\
\text { Energy }(\mathrm{kcal} / \mathrm{mol})\end{array}$} \\
\hline & $(\mathrm{PR})$ & (RT) & (IN) & (PR) & (RT) & (IN) & $(\mathrm{PR})$ & (RT) & (IN) \\
\hline C0M1 & 20.7461 & 27.5109 & 27.6537 & 5.97 & 5.30 & 4.52 & 5.60 & 4.85 & 4.24 \\
\hline C0M2 & 35.4243 & 39.2116 & 30.6883 & 5.85 & 5.69 & 4.78 & 5.85 & 5.59 & 4.78 \\
\hline C0M3 & 28.2399 & 33.6503 & 33.6675 & 4.94 & 4.92 & 3.86 & 4.32 & 4.92 & 3.02 \\
\hline C0M4 & 33.9629 & 32.2289 & 24.2112 & 4.90 & 5.01 & 4.10 & 4.90 & 5.01 & 4.01 \\
\hline C0M5 & 36.8508 & 40.0816 & 36.2464 & 6.90 & 5.96 & 6.61 & 6.90 & 5.96 & 6.61 \\
\hline C0M6 & 34.9761 & 38.8528 & 31.8967 & 6.88 & 6.35 & 5.37 & 6.79 & 5.92 & 5.37 \\
\hline C0M7 & 35.4598 & 37.2454 & 37.5719 & 5.77 & 5.92 & 4.50 & 5.77 & 5.92 & 4.40 \\
\hline C0M8 & 35.9169 & 35.0921 & 38.9619 & 7.32 & 6.95 & 5.67 & 7.32 & 6.95 & 5.42 \\
\hline C0M9 & 31.3565 & 26.5351 & 29.8493 & 5.69 & 6.13 & 5.17 & 5.65 & 6.13 & 4.91 \\
\hline
\end{tabular}

${ }^{*}$ Protease $=(\mathrm{PR})$, Reverse Transcriptase $=(\mathrm{RT})$, Integrase $=(\mathrm{IN})$

*-Lowest Binding Energy, -mean binding energy and -CDOCKER Interaction energy = negative energies

Results obtained from flexible docking (CDOCKER) (Table 2, Fig. 2), states that compound C0M5, C0M6, C0M7 and C0M8 shows the best interaction energy towards all HIV-1 enzymes from several designed compounds. C0M5 has been considered as highest ranked compound and C0M7 shows quite less interaction energy than C0M5, but it has a constant expression towards all enzymes and considered as the second highest and the third highest compound is C0M8 as it has significant energy towards all enzymes. C0M6 could also be considered in the race of best compounds based on CDOCKER results, as it has good energy but it expresses in different way towards each HIV-1 enzyme. Comparative analysis of interaction energy for all compounds states from flexible docking approach, is that all compounds show good energy towards reverse transcriptase enzyme, protease enzyme can be ranked after reverse transcriptase and then integrase enzyme.

Rigid docking results state that (Table 2, Fig. 2), C0M5, C0M6 and C0M8 could be considered as the best compounds. C0M5 expressed equally same towards all HIV-1 enzymes in rigid docking and it shows the efficient lowest binding energy and mean binding energy. C0M8 showed the highest binding energy from all compounds designed in rigid docking approach towards protease and reverse transcriptase enzyme, but not very relevant towards integrase enzyme. C0M6 showed significant interactions as compared to other compounds, but it expresses in different way towards each HIV-1 enzymes. Analyzing results obtained from rigid docking for all compounds, it could be stated that compound behaves good toward protease and reverse transcriptase enzyme, but not all compounds show good interaction towards integrase enzyme except C0M5.

Comparative study of results obtained from rigid and flexible docking approaches for designed 
compounds suggest that, there is not much difference in the interaction of each compounds, they behaves in the same manner in both docking approaches (Fig. 2). Compound shows a good energies towards protease and reverse transcriptase enzyme in both approaches and lower interaction energy towards integrase enzyme. For making a statement for the best compound, results for all ligand and their interactions towards each respective is considered, and then it could be stated that C0M5 and C0M8 are the best compounds among all designed molecules.
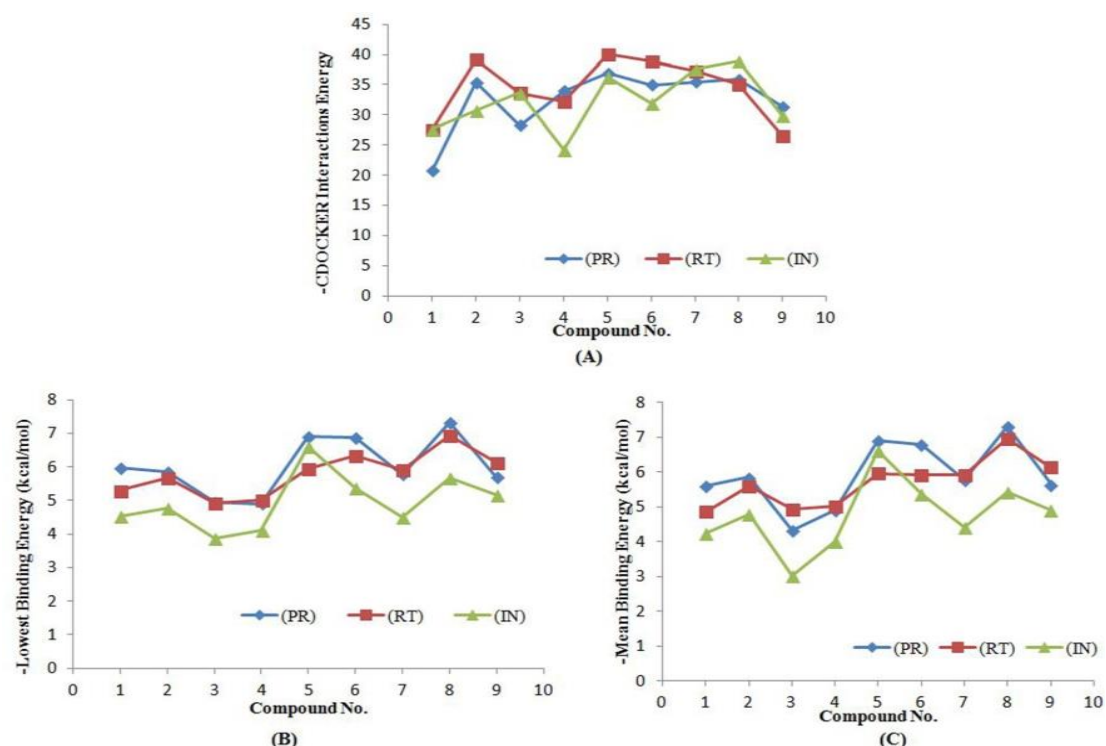

Fig. 2. Interaction/Binding energies of each novel compound towards HIV-1 protease, reverse transcriptase and integrase enzyme. (A) CDOCKER Interaction energy of designed compounds towards respective enzyme obtained through flexible docking; (B) Lowest binding energy obtained from rigid docking using AutoDock of each compounds with respective of HIV-1 enzymes; (C) Mean binding energy obtained from all highest cluster formed using AutoDock (rigid docking) of each compounds with respective of HIV-1 enzymes. (* energy values in graph are negatively represented for PR (Protease), RT (Reverse Transcriptase) and IN (Integrase)).

The best ranked compound C0M5 which has pyridyl methyl piperazine + acetamide at $\mathrm{R}_{1}$ and indanol at $\mathrm{R}_{2}$ end, showed $-36.8508 \mathrm{kcal} / \mathrm{mol}$ CDOCKER Interaction energy and $-6.90 \mathrm{kcal} / \mathrm{mol}$ lowest binding energy (AutoDock) towards HIV-1 protease enzyme, $-40.0816 \mathrm{kcal} / \mathrm{mol}$ CDOCKER Interaction energy and -5.96 $\mathrm{kcal} / \mathrm{mol}$ lowest binding energy (AutoDock) towards HIV-1 reverse transcriptase enzyme and -36.2464 $\mathrm{kcal} / \mathrm{mol}$ CDOCKER Interaction energy and $-6.61 \mathrm{kcal} / \mathrm{mol}$ lowest binding energy (AutoDock) towards HIV-1 integrase enzyme. These results explain the situation that it expresses equally towards each HIV-1 enzymes and also in different docking approaches. Compound that could ranked after C0M5 is C0M8 which has at $\mathrm{R}_{1}$ end, cyclopentenopyridine $+\mathrm{N}$-methylacetamide and at $\mathrm{R}_{2}$ end, octahydro- $1 \mathrm{H}$-isochromene shows almost same condition of showing well defined interactions with HIV-1 enzymes.

Interaction analysis of one of the best compound C0M5 (Fig. 3 and 4) suggests that both different methodologies support each other (COM5 behaves same in terms of interactions). Analyzing interactions towards each enzyme of C0M5, it has been clearly observed that ligands has to generate a very different conformations each time in a cluster to have a good fit with enzyme, this is due to the complexity of HIV-1 enzymes structures, which makes a challenging task to inhibit the activity of these enzymes.

Analyzing compound C0M5 interactions in flexible docking with the HIV-1 protease enzyme (Fig. 3), shows that is forms pi interaction with Leu23 (active site residue) and Arg8 (active site residue) amino acids which are very closely situated with Gly27 (active site residue), in rigid docking ligand form h-binding 
interaction with Thr26 which is bound to Gly27 (active site residue) (Fig. 4). Considering the interaction of C0M5 with HIV-1 reverse transcriptase enzyme, in flexible docking it form h-bond interaction with Lys219 (active site residue) and pi interaction with Arg72 (active site residue), where as in rigid docking it binds with Thr403 by forming hydrogen bond. This is due to the complexity of the reverse transcriptase enzyme. C0M5 forms h-bond interactions with Gln62 a active site residue of HIV-1 integrase enzyme in flexible docking, and in rigid docking is forms h-bond interaction with His67 which is a active site residue of integrase enzyme and Asn117 residue. These interactions states the both methodology supports each other in terms of interactions, and compounds identifies the active site residues of each HIV-1 enzymes and binds efficiently with them.

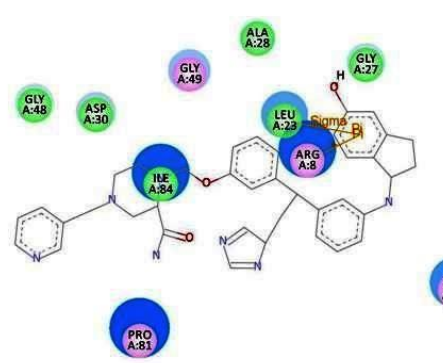

(A)

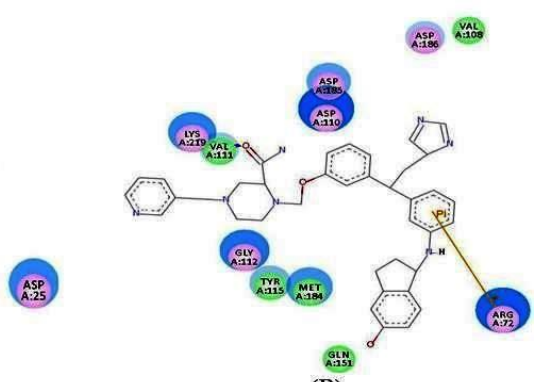

(B)

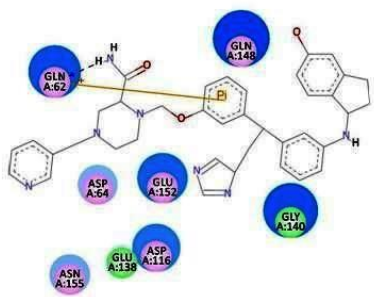

(C)

Fig. 3. Interaction analysis obtained for COM5 with HIV-1 enzymes from CDOCKER (flexible docking) approach. (A) C0M5 interactions with HIV-1 protease; (B) COM5 interactions with HIV-1 reverse transcriptase; (C) C0M5 interactions with HIV-1 integrase. (Color scheme: (A), (B) and (C), carbon atoms are represented in gray color, oxygen atoms in red and hydrogen atoms in white.)

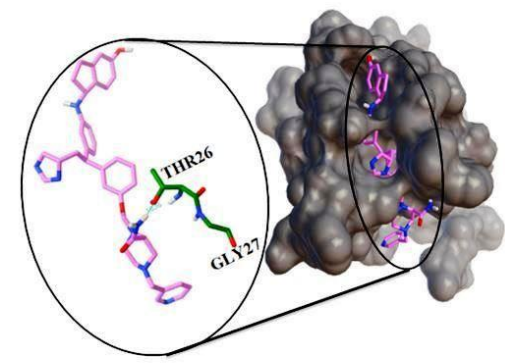

(A)

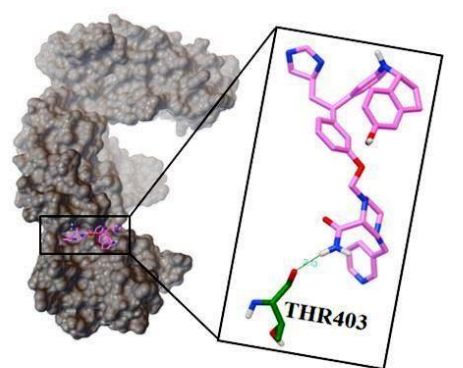

(B)

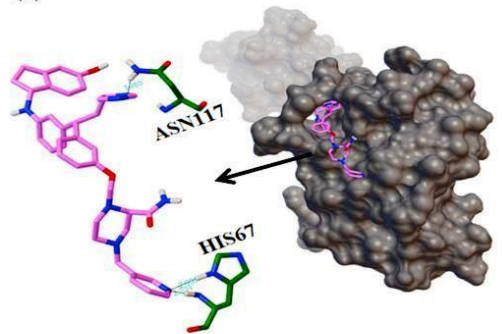

(C)

Fig. 4. Interactions obtained of C0M5 with HIV-1 enzymes from AutoDock (rigid docking) approach. (A) C0M5 interactions with HIV-1 protease; (B) C0M5 interactions with HIV-1 reverse transcriptase; (C) C0M5 
interactions with HIV-1 integrase. (Color scheme: (A), (B) and (C), carbon atoms are represented as pink for ligand and green for amino acids, oxygen atoms in red, nitrogen in blue and hydrogen atom in white.)

\section{Conclusion}

In conclusion, a reverse design approach which was performed to design new compounds, adding functional groups from already available inhibitors to novel scaffold and improving its potency towards HIV-1 protease, reverse transcriptase and integrase resulted into a successful one, designed compound performed as multifunctional ligands. Compounds showed well established interactions in both rigid and flexible docking methods. Although of having a high complexity of HIV-1 enzymes structures, designed ligands had potential interactions with HIV-1 protease, reverse transcriptase and integrase, when making a comparison among these enzymes-ligand interactions HIV-1 integrase is ranked last after other two enzymes.

Considering some artifacts that might coming out from these computational approaches and chemical feasibility of each compounds, the best compound that could ranked in this work is C0M5 and C0M8. In flexible docking compound C0M5 which have pyridyl methyl piperazine with acetamide at $\mathrm{R}_{1}$ end and indanol at $\mathrm{R}_{2}$ end, showed average $-37.7262 \mathrm{kcal} / \mathrm{mol}$ CDOCKER interaction and C0M8 which has at $\mathrm{R}_{1}$ end cyclopentenopyridine with $\mathrm{N}$-methylacetamide and at $\mathrm{R}_{2}$ end octahydro- $1 \mathrm{H}$-isochromene showed average -36.6569 kcal/mol CDOCKER interaction towards HIV-1 protease, reverse transcriptase enzyme and integrase enzymes. In rigid docking, C0M5 has the average lowest binding energy $-6.49 \mathrm{kcal} / \mathrm{mol}$ and identical average mean binding, C0M8 has the average lowest binding energy $-6.64 \mathrm{kcal} / \mathrm{mol}$ and average mean binding energy $-6.56 \mathrm{kcal} / \mathrm{mol}$.

A reverse design approach to design new compounds, a novel scaffold designed for HIV-1 enzymes, examining the multifunctional ligands and testing designed compounds with flexible and rigid docking, were novel areas of this work. Highly ranked compounds C0M5 and C0M8 were designed based on the functional groups of some already available drugs, and they could be considered for further experimental studies to verify results obtained from this work.

\section{Acknowledgment}

Authors are thankful to Prof. Maciej Baginski for sharing his knowledge, and to Supercomputing and Networking Center at Wroclaw University of Technology and Academic Computer Center Cyfronet, Krakow, for providing their hardware and software resources on which rigid docking simulations were calculated.

\section{References}

[1] Nielsen, M., Pedersen, S., \& Kjems, J. (2005). Molecular strategies to inhibit HIV-1 replication. Retrovirology, 2, 10.

[2] Clercq, E. (1998). Toward improvement anti-HIV chemotherapy: therapeutic strategies for intervention with HIV-1 infections. Journal of Medicinal Chemistry, 38, 2491-2517.

[3] Pani, A., Loi, G., Mura, M., Marceddu, T., La Colla, P., \& Marongiu, M. (2002). Targeting HIV: old and new players. Current Drug Targets - Infectious Disorders, 2(1), 17-32.

[4] Coffin, M., Hughes, H., Varmus, E., \& Brown, P. (1997). Integration in Retroviruses. New York: Cold Spring Harbor Laboratory Press.

[5] Pearl, H., \& Taylor, A. (1987). A structural model for the retroviral proteases. Nature, 329, 351-354.

[6] Darke, P., Nutt, R., Brady, S., Garsky, V., Ciccarone, T., Leu, T., et al. (1988). HIV-1 protease specificity of peptide cleavage is sufficient for processing of gag and pol polyproteins. Biochemical and Biophysical Research Communications, 156, 297-303. 
[7] Oroszlan, S., \& Luftig, R. (1990). Retroviral proteinases. Current Topics in Microbiology and Immunology, $157,153-185$.

[8] Schinazi, F., Hernandez-Santiago, I., \& Hurwitz, J. (2006). Pharmacology of current and promising nucleosides for the treatment of human immunodeficiency viruses. Erratum in Antiviral Research, 71, 322-334.

[9] Young, B., \& Kuritzkes, D. (2002). Resistance to HIV-1 protease inhibitors. Infectious Diseases and Therapy, 25, 257-282.

[10] Bacheler, L. (1999). Resistance to non-nucleoside inhibitors of HIV-I reverse transcriptase. Drug Resistance Updates, 2(1), 56-67.

[11] Morphy, R., \& Rankovic, Z. (2009). Designing multiple ligands-medicinal chemistry strategies and challenges. Current Pharmaceutical Design, 15(6), 587-600.

[12] Suri, M., \& Mahmoud, S. (2014). Dual acting HIV inhibitors: integrated rational in silico design strategy, Medicinal Chemistry Research, 23, 682-689.

[13] Wlodawer, A., Miller, M., Jaskolski, M., Snthyanarayana, B., Baldwin, E., Weber, I., et al. (1989). Conserved folding in retrovirial proteases: Crystal structure of a synthetic HIV-1 protease. Science, 245, 616-621.

[14] Kožíšek, M., Lepšík, M., Šašková, K. G., Brynda, J., Konvalinka, J., \& Řezáčová, P. (2014). Thermodynamic and structural analysis of HIV protease resistance to darunavir analysis of heavily mutated patient-derived HIV-1 proteases. FEBS Letters, 281, 1834-1847.

[15] Tu, X., Das, K., Han, Q., Bauman, J., Clark, D., Hou, X., et al. (2010). Structural basis of HIV-1 resistance to AZT by excision. Nature Structural \& Molecular Biology, 17, 1202-1209.

[16] Chen, C., Krucinski, J., Miercke, J., Finer-Moore, S., Tang, H., Leavitt, D., et al. (2000). Crystal structure of the HIV-1 integrase catalytic core and C-terminal domains: a model for viral DNA binding. Proc. Natl. Acad. Sci. USA, 97, 8233-8238.

[17] Barry, C., Mathieu, M., Andrea, F., Yves, P., \& Stephen, H. (2013). A homology model of HIV-1 integrase and analysis of mutations designed to test the model. Journal of Molecular Biology, 425, 2133-2146.

[18] Barbaro, G., Scozzafava, A., Mastrolorenzo, A., \& Supuran, C. (2005). Highly active antiretroviral therapy: current state of the art, new agents and their pharmacological interactions useful for improving therapeutic outcome. Current Pharmaceutical Design, 11, 1805-1843.

[19] Ghosh, K., \& Anderson, D. (2011). Tetrahydrofuran, tetrahydropyran, triazoles and related heterocyclic derivatives as HIV protease inhibitors. Future Medicinal Chemistry, 3(9), 1181-1197.

[20] Brooks, B., Brooks, C., Mackerell, A., Nilsson, L., Petrella, J., Roux, B., et al. (2009). The biomolecular simulation program. Journal of Computational Chemistry, 30, 1545-1615.

[21] Brooks, B., Bruccoleri, R., Olafson, B., States, D., Swaminathan, S., \& Karplus, M. (1983). CHARMM: A program for macromolecular energy, minimization, and dynamics calculations. Journal of Computational Chemistry, 4, 187-217.

[22] Guosheng, W., Robertson, H., Brooks, L., \& Vieth, M. (2003). Detailed analysis of grid-based molecular docking: A case study of CDOCKER-A CHARMm-based MD docking algorithm. Journal of Computational Chemistry, 24, 1549-1562.

[23] Goodsell, D., \& Olson, J. (1990). Automated docking of substrates to proteins by simulated annealing. Proteins: Structure, Function and Genetics, 8, 195-202.

[24] Goodsell, D., Morris, G., \& Olson, A. (1996). Automated docking of flexible ligands: Applications of AutoDock, Journal of Molecular Recognition, 9, 831-835.

[25] Huey, R., Goodsell, D., Morris, M., \& Olson, A. (2004). Grid-based hydrogen bond potentials with improved directionality. Letters in Drug Design \& Discovery, 1, 178-183. 
[26] Morris, G., Goodsell, S., Halliday, S., Huey, R., Hart, E., Belew, K., et al. (1998). Automated docking using a Lamarckian genetic algorithm and empirical binding free energy function. Journal of Computational Chemistry, 19, 1639-1662.

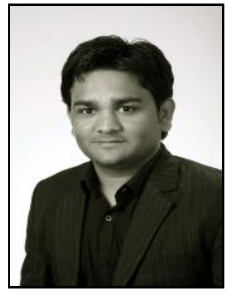

Umesh Kalathiya is currently a doctoral student at the Department of Pharmaceutical Technology and Biochemistry (Faculty of Chemistry), Gdansk University of Technology, Poland. His research is focused on studying receptor-ligand interactions of different complexes. His postgraduate study was focused on molecular docking/modelling methods and completed the M.Sc. degree in 2012 from the Biotechnology (Bioinformatics) Department, Faculty of Chemistry, Wroclaw University of Technology, Poland and graduated in 2010 from Saurashtra University, India with training in database/tool development.

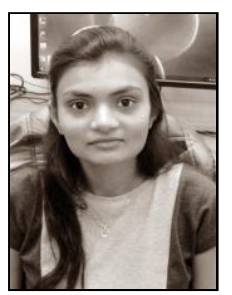

Monikaben Padariya is a full-time doctor at the Department of Pharmaceutical Technology and Biochemistry, Faculty of Chemistry, Gdansk University of Technology (GUT), Poland. Her doctoral research concerns an analysis of multidrug membrane transporters and studies are aimed to overcome the phenomenon of multidrug resistance. Her researches are mainly focused on molecular modelling and molecular docking. Her postgraduate training was in 2012 and completed her M.Sc. degree from the Biotechnology (Bioinformatics) Department, Faculty of Chemistry, Wroclaw University of Technology, Poland and finished her graduate training at Saurashtra University, India in 2010. Her research interest is particularly concerned to study biological systems using molecular modelling, docking and simulation techniques. 